\title{
Glomus tumor of the anterior urethra: A rare case report and review of the literature
}

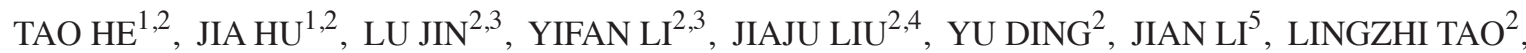 \\ ZEBO CHEN $^{2}$, LIANGCHAO NI ${ }^{2}$, SHANGQI YANG ${ }^{2}$, XIANGMING MAO $^{2}$ and YONGQING LAI ${ }^{2}$ \\ ${ }^{1}$ Department of Urology, Guangzhou Medical University, Guangzhou, Guangdong 511436; ${ }^{2}$ Department of Urology, \\ Peking University Shenzhen Hospital, Shenzhen, Guangdong 518036; ${ }^{3}$ Department of Urology, Anhui Medical University, \\ Hefei, Anhui 230032; ${ }^{4}$ Department of Urology, Shantou University Medical College, Guangdong 515041; \\ ${ }^{5}$ Department of Pathology, Peking University Shenzhen Hospital, Shenzhen, Guangdong 518036, P.R. China
}

Received September 28, 2015; Accepted March 2, 2016

DOI: $10.3892 / \mathrm{mco} .2016 .832$

\begin{abstract}
Glomus tumors are rare and benign neoplasms, which normally originate from peripheral soft tissue. To date, reported cases of glomus tumor occurring in genitourinary tract, particularly in the urethra, are exceedingly rare. The present study presented a rare case of glomus tumor of the anterior urethra in a 42 year-old male, his main complaints were a history of anterior urethra pain for 3 years, and a palpable and tender mass in the urethra for 2 weeks. Urethrocystoscopy examination and the resection of the urethral mass were performed. Pathological and immunohistochemical examination revealed that the mass was a benign glomus tumor. The patient remained in good condition by 6 month follow-up, and revealed no problems or recurrence following surgery. This is the first case, to the best of our knowledge, to present a glomus tumor occurring in a male's urethra and the present report provided a supplementary review for the previous cases and the literature.
\end{abstract}

\section{Introduction}

Glomus tumors, which consist of modified smooth muscle cells, are a type of mesenchymal neoplasm arising in the extremities, which are filled with plenteous glomus bodies most of time, and the most typical region is the subungual region of the fingers (1). The onset of a glomus tumor is rare, which accounts for no more than $2 \%$ of soft tissue neoplasms with an equal incidence in genders $(2,3)$. Glomus bodies have a thermoregulation function, as its vascular components are composed of afferent arteriole, efferent venule and anastomotic Suquet-Hoyer canal (4). A previous genetic study found out that certain cases are correlated with the truncating mutations on chromosome 1p21-22 (1). The majority of glomus tumors are benign, while patients diagnosed

Correspondence to: Professor Yongqing Lai, Department of Urology, Peking University Shenzhen Hospital, 1120 Lianhua Road, Shenzhen, Guangdong 518036, P.R. China

E-mail: yqlord@163.com

Key words: glomus tumor, urethra, differential diagnosis with malignant glomus tumors accounted for no more than $1 \%$ of all glomus tumors (5).

To date, glomus tumors have been reported outside of the usual peripheral soft tissue regions, including the gastrointestinal tract (6), liver (7), lung (8) and pancreas (9). Cases reported in genitourinary tract are rare, and the majority cases among those reports are clitoris, labia, cervix, vagina, bladder, penis (3) and kidney (2). However, reports of glomus tumor of the urethra are extremely rare. When searching PubMed and reviewing the previous literature, only three cases reported in the periurethral tissue of female exist, and most of which were located in the periurethral area of the vulva. The characters of each case are shown in Table I $(4,10,11)$.

The present study presented a rare benign glomus tumor of the anterior urethra in a 42 year-old male. This male remains in good condition following surgical resection of the urethral mass. In addition, to the best of our knowledge, this is the first case of glomus tumor occurring in a male's anterior urethra. The present study was approved by the Ethics Committee of Peking University Shenzhen Hospital (Shenzhen, China) and the written informed consent was obtained from the patient.

Case report. A 42 year-old male attended the outpatient department of Shenzhen Hospital with dominating complaints of a history of anterior urethra pain for 3 years and a mass with tenderness in the urethra for 2 weeks. The patient was subsequently admitted to the Department of Urology at Peking University Shenzhen Hospital for further examination and therapy. The patient exhibited a symptom of mild dysuria, however, no frequency, urgency and hematuria. Furthermore, no respiratory, cardiovascular, or constitutional symptoms were exhibited. A previous history of hypertension, diabetes, tuberculosis, hepatitis or surgery were denied. Physical examination revealed a male in good condition with regards to development and nutrition, abnormal signs were not found, with the exception of a firm and tender mass, which was $1 \times 1 \mathrm{~cm}$ in size, which was palpated in the anterior urethra. Laboratory examinations, including routine hematological tests and biochemical examination were all within the normal ranges.

Urethrocystoscopy examination and the resection of the urethral mass were performed on the fifth day post-admission. 
Table I. Reported cases of periurethral glomus tumors.

\begin{tabular}{|c|c|c|c|c|c|c|c|}
\hline Author, year & $\begin{array}{l}\text { Ages } \\
\text { (years) }\end{array}$ & Gender & Clinical features & $\begin{array}{l}\text { Tumor size } \\
\qquad(\mathrm{cm})\end{array}$ & Location & Immunohistochemistry & Refs \\
\hline Malowany et al, 2008 & 61 & Female & $\begin{array}{l}\text { Focally ulcerating, } \\
\text { soft periurethral mass }\end{array}$ & NA & Periurethral area & $\begin{array}{l}\operatorname{Vimentin}(+), \operatorname{desmin}(+), \\
\text { calponin }(+)\end{array}$ & (4) \\
\hline Sonobe et al, 1994 & 46 & Female & $\begin{array}{l}\text { Painful nodule } \\
\text { of the vulva }\end{array}$ & 2 & Periurethral area & Vimentin(+), SMA(+) & (10) \\
\hline Blandamura et al, 2000 & 26 & Female & $\begin{array}{l}\text { Painful nodule } \\
\text { (submucosal tissue } \\
\text { of the vulva) }\end{array}$ & 5 & Periurethral area & Vimentin(+), SMA(+) & (11) \\
\hline
\end{tabular}

SMA, smooth muscle actin; NA, not available.
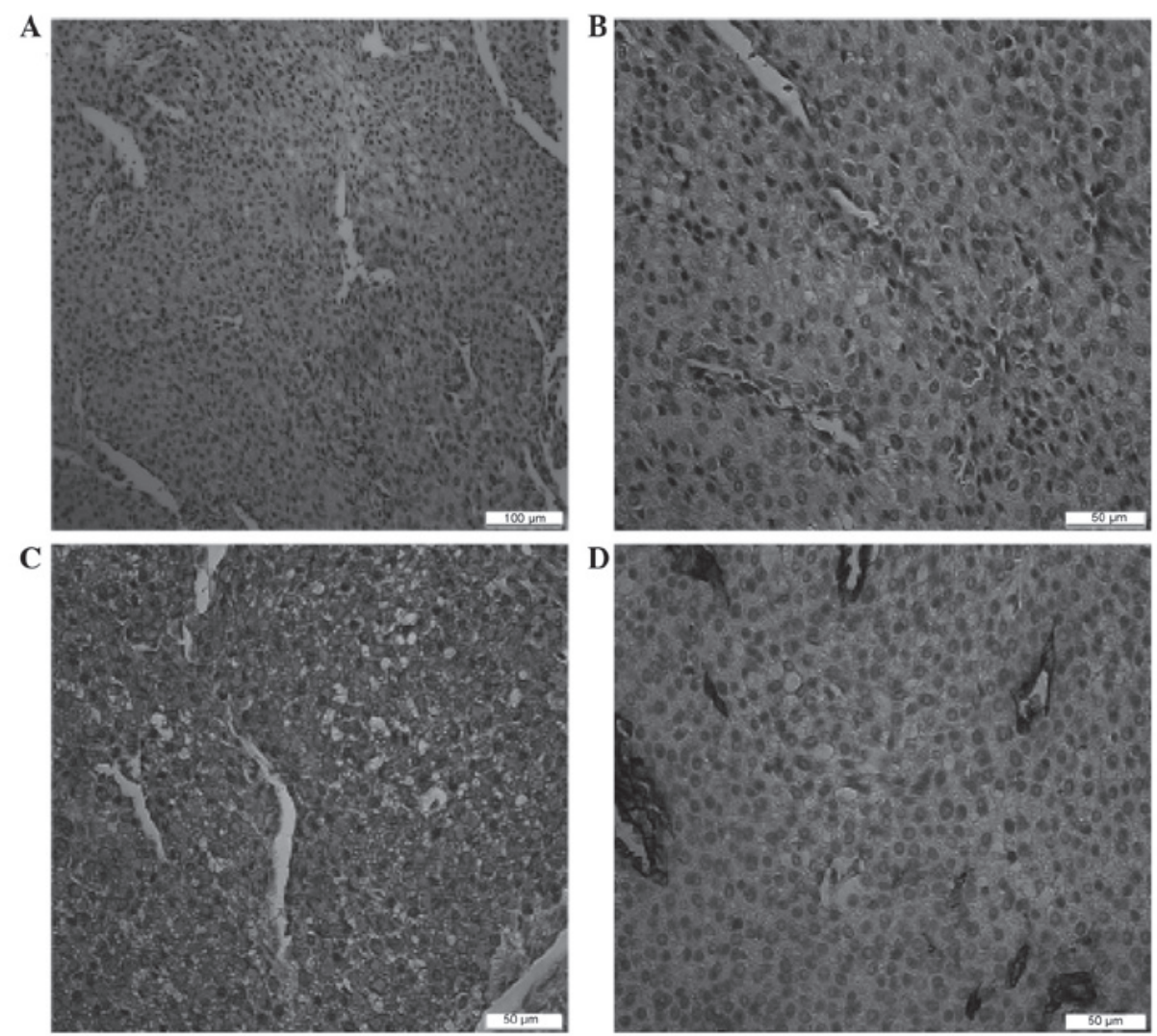

Figure 1. Pathological and immunohistochemical examination. (A and B) Pathological examination revealed that the tumor tissues contained abundant vessels, surrounded by the tumor cells, which exhibited a uniform appearance, with a eosinophilic cytoplasm. The nuclei were a moderate size, round to oval and of a regular shape (magnification, x200 and x400, respectively). The immunohistochemical staining revealed that the tumor cells were positive for (C) smooth muscle actin and (D) cluster of differentiation 31 (magnification, x400). Scale bar: (A) $100 \mu \mathrm{m}$; (B-D) $50 \mu \mathrm{m}$.

A mass with complete envelope, $1 \mathrm{~cm}$ in diameter and firmly located in the cavernous body of urethra, was observed and the mass was subsequently excised. Pathological examination revealed that the tumor tissues contained abundant vessels, surrounded by the tumor cells, which exhibited a uniform appearance with an eosinophilic cytoplasm, and the nuclei were a moderate size, round to oval and regular shaped (Fig. 1A and B). Immunohistochemical analysis revealed that the tumor cells were positive for smooth muscle actin (SMA; Fig. 1C) and cluster of differentiation (CD)31 (vessel endothelium; Fig. 1D), while negative for desmin.
The result of the cell proliferation marker, Ki-67, was $\sim 2-3 \%$. The diagnosis of glomus tumor of the anterior urethra was confirmed.

The patient was discharged on the fifth postoperative day. Follow-up at 6 months revealed no problems or signs of recurrence.

\section{Discussion}

Glomus tumors are uncommon mesenchymal neoplasms: A previous study revealed that they may result from the 
hyperplasia or overgrowth of the glomus bodies (3). The most common symptoms normally present as intermittent painful, red to blue and tender nodules in the subcutaneous regions (1). According to the proportions of their components, glomus tumors are usually divided into three subgroups, including glomus tumor proper, glomangiomyoma and glomangioma (12). A glomus tumor of the urinary tract is extremely rare. The present study reported the first case, to the best of our knowledge, of a glomus tumor of the urethra in a male.

As most of the results of magnetic resonance imaging in glomus tumors are dark lesions on T1-weighted and bright, high intensity lesions on T2-weighted images, it may be helpful for the diagnosis of glomus tumors (13). However, the pathological and immunohistochemical examination are the standard in diagnosing glomus tumors. Typical features of glomus tumors in pathology are as follows: Plenteous vascular surrounded by uniformly round to oval tumor cells, exhibiting an eosinophilic cytoplasm with well-defined margins, and the nuclei are uniform with bland chromatin and with inconspicuous to well-demarcated nucleoli (1). Immunohistochemical examination revealed that the glomus cells have a strong positive immunoreaction with the SMA and vimentin, however, are normally negative for vascular endothelial markers, including CD31 or CD34, epithelial markers, including cytokeratin, and neuroendocrine markers, however, desmin is alterable $(2,12)$.

In the present case, the tumor cells were positive for CD31, while negative for desmin, and the result of the cell proliferation marker, Ki-67, was $\sim 2-3 \%$. Notably, SMA staining was positive. However, of the three previous reported cases of periurethral glomus tumor, Malowany et al (4) revealed that the tumor cells were positive for desmin and vimentin, but negative for SMA. It is therefore important to pay attention to those patients with glomus tumors who present a negative immunoreaction with SMA or CD31 in order to avoid a missed diagnosis.

It is important to differentiate glomus tumors with other neoplasms, including hidradenoma, angiomyolipoma, hemangiopericytoma, juxtaglomerular tumor, renal cell carcinoma, epithelioid leiomyoma, carcinoid tumor and paraganglioma of the genitourinary tract, to avoid unnecessary examinations and treatments. Each neoplasm has its own immunohistochemical staining feature: Compared with a glomus tumor, hidradenoma is positive for cytokeratin, carcinoembryonic antigen and epithelial membrane antigen; juxtaglomerular tumor can cause hypertension in young adults due to its over-secretion of rennin; hemangiopericytoma is positive for vascular endothelial markers, including CD34, but is negative for SMA; angiomyolipoma presents a strong immunoreaction with HMB-45 $(1,2,12,14)$. Additionally, when it comes to a glomus tumor of the urethra, periurethral or urethral non-neoplastic masses and lesions, differential diagnosis must be taken into consideration, which include urethral diverticulum, prolapsed ectopic ureterocele, urethral prolapse, pseudocyst and granulomatous inflammation of urethra (4).

Although malignant glomus tumors are extremely rare, accurate diagnosis of benign or malignant glomus tumors is of great important since the metastasizing risk is up to $25 \%$ (1). According to the histopathological criteria of malignant glomus tumors (15), the glomus tumor reported in our case was finally confirmed as a benign glomus tumor of the anterior urethra, and no problems and recurrent signs were reported at the 6 month follow-up.

In conclusion, glomus tumors of the genitourinary tract are exceedingly rare. A rare number of cases of glomus tumors of the periurethra have been reported previously. The present study reported for the first time, to the best of our knowledge, the first case of a glomus tumor of the anterior urethra in a male. Pathological and immunohistochemical examination can establish a definitive diagnosis and assist with differentiating malignant glomus tumors or other neoplasms. Complete resection of the tumor mass is a efficient treatment and can avoid local recurrence. As malignant glomus tumors are exceedingly rare, further studies on malignant glomus tumors and the survival rate are required.

\section{Acknowledgements}

The present study was supported by the National Natural Science Foundation of China (no. 81101922), Science and Technology Development Fund Project of Shenzhen (nos. JCYJ20130402114702124 and JCYJ2015040309 1443329) and the fund of Guangdong Key medical subject.

\section{References}

1. Gombos Z and Zhang PJ: Glomus tumor. Arch Pathol Lab Med 132: 1448-1452, 2008.

2. Sugimoto K, Yamamoto Y, Hashimoto K, Esa A and Okamoto S: Glomus tumor of the kidney. Int J Urol 17: 187-188, 2010.

3. Tripodi SA, Rocca BJ, Mourmouras V, Barbanti G, Colecchia M and Ambrosio MR: Benign glomus tumor of the urinary bladder. Arch Pathol Lab Med 137: 1005-1008, 2013.

4. Malowany JI, Rieckenberg RM, Okafo BA and Colgan TJ: Glomus tumor presenting as a periurethral mass. J Low Genit Tract Dis 12: 316-319, 2008.

5. Shim HS, Choi YD and Cho NH: Malignant glomus tumor of the urinary bladder. Arch Pathol Lab Med 129: 940-942, 2005.

6. Miettinen M, Paal E, Lasota J and Sobin LH: Gastrointestinal glomus tumors: A clinicopathologic, immunohistochemical and molecular genetic study of 32 cases. Am J Surg Pathol 26: 301-311, 2002.

7. Jaiswal VR, Champine JG, Sharma S and Molberg KH: Primary glomangioma of the liver: A case report and review of the literature. Arch Pathol Lab Med 128: e46-e49, 2004

8. Baena-Del Valle JA, Murillo-Echeverri VE, Gaviria-Velásquez A, Celis-Mejía DM and Matute-Turizo G: Glomus tumour of the lung: A case report and literature review. Cir Cir 83: 319-323, 2015 (In Spanish).

9. Miliauskas JR, Worthley C and Allen PW: Glomangiomyoma (glomus tumour) of the pancreas: A case report. Pathology 34: 193-195, 2002.

10. Sonobe H, Ro JY, Ramos M, Diaz I, Mackay B, Ordonez NG and Ayala AG: Glomus tumor of the female external genitalia: A report of two cases. Int J Gynecol Pathol 13: 359-364, 1994.

11. Blandamura S, Florea G, Brotto M, Salmaso R and Castellan L: Periurethral glomangiomyoma in women: Case report and review of the literature. Histopathology 36: 571-572, 2000.

12. Onishi T, Kato M and Hoshina A: Glomus tumor of the hypoplastic kidney. Int J Urol 17: 677-678, 2010.

13. Al-Qattan MM, Al-Namla A, Al-Thunayan A, Al-Subhi F and El-Shayeb AF: Magnetic resonance imaging in the diagnosis of glomus tumours of the hand. J Hand Surg Br 30: 535-540, 2005.

14. Herawi M, Parwani AV, Edlow D, Smolev JK and Epstein JI: Glomus tumor of renal pelvis: A case report and review of the literature. Hum Pathol 36: 299-302, 2005.

15. Khoury T, Balos L, McGrath B, Wong MK, Cheney RT and Tan D: Malignant glomus tumor: A case report and review of literature, focusing on its clinicopathologic features and immunohistochemical profile. Am J Dermatopatho 27: 428-431, 2005. 